SNUTP97/032

\title{
Strange Form Factors of Baryons
}

\author{
Soon-Tae Hong ${ }^{a}$, Byung-Yoon Park ${ }^{b}$ and Dong-Pil Min ${ }^{a}$ \\ ${ }^{a}$ Department of Physics and Center for Theoretical Physics, \\ Seoul National University, Seoul 151-742, Korea \\ ${ }^{b}$ Department of Physics, \\ Chungnam National University, Daejeon 305-764, Korea
}

September 13, 2018

\begin{abstract}
The strange magnetic form factor of proton is calculated in a model independent way to confirm the recent experimental result of the SAMPLE Collaboration. We consider a set of six inertia parameters to realize the magnetic moments of the baryon octet. We show that the strange form factor of proton is a positive quantity, i.e. +0.37 n.m. Its positiveness is analyzed in terms of the vacuum fluctuation coupled to the vector current along the strangeness direction.
\end{abstract}

PACS; 21.60; 13.40.G

Keywords; strange form factors; chiral bag 
Triggered by the EMC result[1], there have been significant discussions concerning the possibility of sizable strange quark matrix elements in the nucleon. Quite recently, the SAMPLE experiment [2] reported the proton's neutral weak magnetic form factor, which has been suggested by the neutral weak magnetic moment measurement through parity violating electron scattering[3]. In fact, if the strange quark content in the nucleon is substantial then kaon condensation can be induced at a matter density lower than that of chiral phase transition [4, 5] affecting the scenarios for relativistic heavy-ion reactions [6], neutron star cooling [7] and so on.

On the other hand, McKeown $[8]$ has shown that the strange form factor of proton should be positive by using the conjecture that the up-quark effects are generally dominant in the flavor dependence of the nucleon properties. This result is contrary to the negative values of the proton strange form factor which result from most of the model calculations 9, 10, 11, 12, 13, 14] except that of Hong and Park (HP) [15] based on the SU(3) chiral bag model (CBM) 16]. Recently Meissner et al investigated the strange form factors by taking isoscalar and isovector meson poles into account [17]. They found the strange form factor to be positive and quite "OZI-resurrected", $\mu_{s} \approx 0.003$ n.m..

The motivation of this paper is to justify the prediction of Ref. 115] on the positive strange form factor of the proton by doing the adjustment of the inertia parameters in a more systematic way.

Long ago, Adkins discussed the model independent parameterization of the magnetic moments of baryons governed by the group structure, in which the flavor symmetry breaking and the $1 / N_{c}$ correction are taken into account of the SU(3) Skyrme model (SM) 18]. The same line was traced in HP for the CBM to show that there are also six parameters for the magnetic moments of baryon octet. As the details of the calculation with CBM already are presented in HP, we here mention only the general scheme of it to discuss the modelindependent behavior of the magnetic moments.

In the CBM, a baryon is described by two phases : the mesonic phase of soliton configuration is surrounding the quark phase where the freely moving quarks are confined through the chirally symmetric boundary condition. In addition to the "chirally symmetric" Lagrangian, incorporated are various "symmetry breaking" terms into the Lagrangian: (1) Breaking of the $\mathrm{SU}(3)_{L} \times \mathrm{SU}(3)_{R}$ chiral symmetry into $\mathrm{SU}(3)_{V}$ for the nonvanishing quark masses. At this level all the quark masses and consequently the masses of the meson octet are chosen degenerate. The mass matrix $\bar{M}$ is given by $\bar{M}=\bar{m} \operatorname{diag}(1,1,1)$ with $\bar{m}=\frac{1}{2}\left(m_{u}+m_{d}\right)$. (2) Breaking of the flavor $\mathrm{SU}(3)$ symmetry into $\mathrm{SU}(2) \times \mathrm{U}(1)$ due to the heavier strange quark than the other two light quarks and due to the difference in the kaon and pion decay constants $f_{K} \neq f_{\pi}$. The isospin symmetry breaking has not been incorporated. These symmetry breaking terms can be treated as perturbations.

The meson part of the solution is described by a classical static field configuration $U_{0}(\vec{r})=\exp \left(i \sum_{i=1}^{3} \lambda_{i} \hat{r}_{i} \theta(r)\right)$ and the quark part is described by a Fock state $|H\rangle_{0}$ with $N_{c}$ valence quarks and completely filled negative energy sea for the quarks confined inside the bag. The zero modes associated with invariance of the solution under the arbitrary rotations in $\mathrm{SU}(3)$ flavor space can be canonically quantized by introducing proper collective coordinates $A(t) \in \mathrm{SU}(3)$. Such a process leads us to the Hamiltonian for the baryon states as

$$
\hat{H}=\hat{H}_{0}+\hat{H}_{S B},
$$


where

$$
\hat{H}_{0}=M_{0}+\frac{1}{2}\left(\frac{1}{\mathcal{I}_{1}}-\frac{1}{\mathcal{I}_{2}}\right) \hat{J}^{2}+\frac{1}{2 \mathcal{I}_{2}}\left(\hat{C}_{2}^{2}-\frac{3}{4} \hat{Y}_{R}^{2}\right)
$$

and

$$
\hat{H}_{S B}=m\left[1-\hat{D}_{88}^{8}(A)\right]-m_{1} \hat{Y} .
$$

Here, the operators associated with the SU(3) collective coordinate quantization will be distinguished by putting a caret : $\hat{C}_{2}^{2}$ is the quadratic Casimir operator for flavor $\mathrm{SU}(3)$, $\hat{J}_{i}(i=1,2,3)$ the spin operator, $\hat{Y}_{R}$ the "right" hypercharge operator, $D_{a b}^{8}(A)$ the adjoint representation of $\mathrm{SU}(3)$, and $\hat{Y}$ the hypercharge operator. The other quantities are the inertia parameters : $M_{0}$ is the mass of the soliton solution, $\mathcal{I}_{1}$ and $\mathcal{I}_{2}$, respectively, are its moments of inertia with respect to the collective rotation in the nonstrangeness and strangeness directions, and $m$ and $m_{1}$ are inertia parameters associated with the flavor symmetry breaking (FSB). (See Ref. [16] for their explicit form.) Except these model dependent inertia parameters, the Hamiltonian of Eq.(11) is general to all the soliton models for baryons based on the chiral Lagrangian.

As far as the symmetric part of the Hamiltonian $H_{0}$ is concerned, the wavefunction of the baryon with isospin $\left(I, I_{3}\right)$, spin $\left(J, J_{3}\right)$ and the hypercharge $Y$ is given by the Wigner $D$-function,

$$
\Phi_{B}^{(\lambda)}(A)=\sqrt{\lambda}\left\langle I, I_{3}, Y\left|D^{(\lambda)}(A)\right| J,-J_{3},+1\right\rangle,
$$

where $D^{(\lambda)}(A)$ is the matrix element of the $\mathrm{SU}(3)$ irreducible representation (IR) of dimension $\lambda$ acting on the basis $\left\langle I, I_{3}, Y\right|$ and $\left|J,-J_{3}, Y_{R}\right\rangle$. Due to the Wess-Zumino constraint, only the states obeying $Y_{R}=1$ are allowed. When the symmetry breaking Hamiltonian $\hat{H}_{S B}$ is treated as a perturbation, up to first order the wavefunctions of the baryon octet are modified as

$$
\Phi_{B}(A)=\Phi_{B}^{(8)}(A)-C_{(\underline{10})} \Phi_{B}^{(\underline{10})}(A)-C_{(27)} \Phi_{B}^{(27)}(A),
$$

where $\Phi_{B}^{(10)}(A)$ and $\Phi_{B}^{(27)}(A)$ have the same quantum numbers of the corresponding baryon belonging to different IR. Such a "representation mixing" is caused by the $\hat{D}_{88}^{8}(A)$ in $\hat{H}_{S B}$.

The baryon magnetic moments can be calculated by taking the expectation values of the corresponding operator with respect to the baryon wavefunctions (3). We first derive the flavor singlet current $V_{\mu}^{(0)}$ and flavor octet vector current $V_{\mu}^{(a)}(a=1,2, \cdots, 8)$ from the given CBM Lagrangian. As for a baryon number current on the mesonic phase, we should include that of the topological winding number. In terms of these current, the electromagnetic (em) currents $J_{\mu}^{e m}$ for example can be easily constructed as $J_{\mu}^{e m}=V_{\mu}^{(3)}+\frac{1}{\sqrt{3}} V_{\mu}^{(8)}$. The currents define associated magnetic moments as

$$
\vec{\mu}^{(0, a)}=\frac{1}{2} \int \mathrm{d}^{3} r\left(\vec{r} \times \vec{V}^{(0, a)}\right) .
$$

Following the standard procedures in the SU(3) collective coordinate quantization scheme, 
one can obtain the magnetic moment operators in terms of the collective variables as

$$
\begin{aligned}
\hat{\mu}^{i(0)}= & \mathcal{M} \hat{J}^{i}, \\
\hat{\mu}^{i(a)}= & -\mathcal{N} \hat{D}_{a i}^{8}-\mathcal{N}^{\prime} \sum_{p, q=4}^{7} d_{i p q} \hat{D}_{a p}^{8} \hat{T}_{q}^{R}+\frac{N_{c}}{2 \sqrt{3}} \mathcal{M} \hat{D}_{a 8}^{8} \hat{J}_{i} \\
& -\mathcal{P} \hat{D}_{a i}^{8}\left(1-\hat{D}_{88}^{8}\right)+\frac{\sqrt{3}}{2} \mathcal{Q} \sum_{p, q=4}^{7} d_{i p q} \hat{D}_{a p}^{8} \hat{D}_{8 q}^{8}
\end{aligned}
$$

where $\mathcal{M}, \mathcal{N}, \mathcal{N}^{\prime}, \mathcal{P}$ and $\mathcal{Q}$ are new inertia parameters depending on the soliton solution. (See Refs. 115, 19] for the details.) $\hat{T}_{p}^{R}(p=4, \cdots, 7)$ is the "right" $\mathrm{SU}(3)$ operator appearing in the $\mathrm{SU}(3)$ collective coordinate quantization processes. The other operators such as $\hat{T}_{i}^{R}(i=1,2,3)$ and $\hat{T}_{8}^{R}$ have been replaced by familiar spin operator $\hat{J}_{i}$ and the right hypercharge operator $Y^{R}$ mentioned before : $\hat{J}_{i}=-\hat{T}_{i}^{R}$ and $\hat{Y}^{R}=2 \sqrt{\frac{1}{3}} \hat{T}_{8}^{R}$. The last two terms of $\hat{\mu}^{i(a)}$ come from the symmetry breaking Lagrangian for the difference in the meson decay constants. In the practical calculations of the expectation values, it is helpful to rewrite the last two expressions in terms of a linear combination of the single $\hat{D}$ operators, for example, as

$$
\begin{aligned}
& \hat{\mu}_{S B}^{i(3)}=\mathcal{P}\left(-\frac{4}{5} \hat{D}_{3 i}^{8}+\frac{1}{4}\left(\hat{D}_{3 i}^{10}+\hat{D}_{3 i}^{\frac{10}{3 i}}\right)+\frac{3}{10} \hat{D}_{3 i}^{27}\right)+\mathcal{Q}\left(\frac{3}{10} \hat{D}_{3 i}^{8}-\frac{3}{10} \hat{D}_{3 i}^{27}\right), \\
& \hat{\mu}_{S B}^{i(8)}=\mathcal{P}\left(-\frac{6}{5} \hat{D}_{8 i}^{8}+\frac{9}{20} \hat{D}_{8 i}^{27}\right)+\mathcal{Q}\left(-\frac{3}{10} \hat{D}_{8 i}^{8}-\frac{9}{20} \hat{D}_{8 i}^{27}\right) .
\end{aligned}
$$

Here, $D_{a b}^{\lambda}$ is the unitary IR of SU(3), which is nothing but the Wigner $D$-functions appearing in Eq.(3) with the corresponding quantum numbers $\left(Y ; I, I_{3}\right)_{a}$ and $\left(Y_{R} ; J,-J_{3}\right)_{b}$.

By taking the expectation values of the operators (5) with the wavefunctions (3) for the baryon states, one can obtain the electromagnetic moments in a form of

$$
\mu_{B}=\mu_{0, B}+\delta \mu_{1, B}+\delta \mu_{2, B}
$$

up to the first order in perturbation. Here, the first order correction $\delta \mu_{1, B}$ comes from the explicit contribution of the FSB Lagrangian to the current and $\delta \mu_{2, B}$ is due to the representation mixing in the wavefunctions. In Table 1 , listed are the explicit expressions for the magnetic moments of the baryon octet. Note that $\delta \mu_{2, B}$ (and the term proportional to $\mathcal{Q}$ in $\left.\delta \mu_{1, B}\right)$ satisfy "V-spin symmetric" relations

$$
\delta \mu_{2, p}=\delta \mu_{2, \Xi^{-}}, \quad \delta \mu_{2, n}=\delta \mu_{2, \Sigma^{-}} \quad \text { and } \quad \delta \mu_{2, \Sigma^{+}}=\delta \mu_{2, \Xi^{0}}\left(=\frac{1}{2} \delta \mu_{2, p}\right),
$$

while $\mu_{0, B}$ 's satisfy $\mathrm{SU}(3)$ symmetric (or "U-spin symmetric") ones

$$
\mu_{0, p}=\mu_{0, \Sigma^{+}}, \quad \mu_{0, n}=\mu_{0, \Xi^{0}}, \quad \mu_{0, \Sigma^{-}}=\mu_{0, \Xi^{-}} \quad \text { and } \quad \mu_{0, \Lambda}=-\mu_{0, \Sigma^{0}} .
$$

At this point, it will be interesting to evaluate the separate up, down and strange quark contributions to the magnetic moments of the baryons. They are associated with the electromagnetic current carried by the quark of flavor $f(=u, d, s)$, which are obtained by multiplying the fractional charge to the corresponding quark current $V_{\mu}^{(f)}$; that is, $J_{\mu}^{e m(f)}=$ 
$Q_{e m}^{(f)} V_{\mu}^{(f)}$ with $Q_{e m}^{(u)}=+\frac{2}{3}, Q_{e m}^{(d, s)}=-\frac{1}{3}$ and

$$
\begin{aligned}
& V_{\mu}^{(u)}=V_{\mu}^{(0)}+V_{\mu}^{(3)}+\frac{1}{\sqrt{3}} V_{\mu}^{(8)}, \\
& V_{\mu}^{(d)}=V_{\mu}^{(0)}-V_{\mu}^{(3)}+\frac{1}{\sqrt{3}} V_{\mu}^{(8)}, \\
& V_{\mu}^{(s)}=V_{\mu}^{(0)}-\frac{2}{\sqrt{3}} V_{\mu}^{(8)} .
\end{aligned}
$$

Let $\mu_{B}^{(f)}$ denote the each quark contribution to the magnetic moment, which can be obtained in the same way described above. The explicit expressions for $\mu_{B}^{(s)}$ are appended in Table 2. Note that the baryons belonging to the same isospin multiplets have the same strange component. The rest up- and down-quark contributions can be obtained with the help of $\mu_{B}=\mu_{B}^{(u)}+\mu_{B}^{(d)}+\mu_{B}^{(s)}$ and by using the isospin symmetry :

$$
\mu_{B}^{(d)}=\frac{Q_{e m}^{(d)}}{Q_{e m}^{(u)}} \mu_{\tilde{B}}^{(u)},
$$

where $\tilde{B}$ denotes for the isospin conjugate baryon to $B$.

The form factors are defined through

$$
{ }_{B}\left\langle p+q\left|V_{\mu}^{(f)}\right| p\right\rangle_{B}=\bar{\psi}_{B}\left[\gamma_{\mu} F_{1, B}^{(f)}\left(q^{2}\right)+\frac{i}{2 m_{B}} \sigma_{\mu \nu} q^{\nu} F_{2, B}^{(f)}\left(q^{2}\right)\right] \psi_{B}
$$

where $\psi_{B}$ is the spinor for the baryons. In the limit of $q^{2} \rightarrow 0, \mu_{B}^{(f)}$ are related to the form factors $F_{2, B}^{(f)}\left(q^{2}\right)$ discussed in the literature. The relation between the form factors $F_{i, B}^{(f)}(i=1,2)$ and the each component of the magnetic moments $\mu_{B}^{(f)}$ reads

$$
\mu_{B}^{(f)}=Q_{e . m}^{(f)}\left[F_{1, B}^{(f)}(0)+F_{2, B}^{(f)}(0)\right]
$$

On the other hand, the form factor $F_{1, B}^{(f)}$ is just the number of valence quarks with the flavor $f$ contained in the baryon, which is trivially given by its quantum numbers as

$$
F_{1, B}^{(u)}(0)=1+I_{3}+\frac{1}{2} Y, \quad F_{1, B}^{(d)}(0)=1-I_{3}+\frac{1}{2} Y, \quad \text { and } \quad F_{1, B}^{(s)}(0)=1-Y .
$$

Thus, the strange quark contributions to the electromagnetic moments can be transformed into those for the $F_{2, B}^{(s)}$ form factors by

$$
\begin{array}{ll}
F_{2, N}^{(s)}(0)=-3 \mu_{N}^{(s)}, & F_{2, \Lambda}^{(s)}(0)=-3 \mu_{\Lambda}^{(s)}-1, \\
F_{2, \Sigma}^{(s)}(0)=-3 \mu_{\Sigma}^{(s)}-1, & F_{2, \Xi}^{(s)}(0)=-3 \mu_{\Xi}^{(s)}-2 .
\end{array}
$$

Note that the strange magnetic moment of nucleon comes solely from the $F_{2, N}^{(s)}(0)$. Furthermore, the I-spin symmetric relation (11) can be expressed in a simpler form as

$$
F_{2, B}^{(u)}(0)=F_{2, \tilde{B}}^{(d)}(0)
$$

We summarize the expressions for the magnetic moments of baryons in Table 1 which are general to various chiral soliton models of baryons. Then, we optimize the inertia parameters 
to fit the data in the point of view that the situation can be improved by fine-tuning the model, for example, by including the other degrees of freedom and/or higher order terms in the derivative on the chiral field. In Ref. [15], this trial was done by varying roughly the inertia parameters around the CBM values to minimize the sum of the absolute difference between the model predictions and the measured magnetic moments of the baryons

In this work, we develop an improved fitting method by noticing that the formulas for the baryon magnetic moments are linear in the inertia parameters except $m \mathcal{I}_{2}$. Thus, given a number for $m \mathcal{I}_{2}$, a standard least-square-fitting method can be applied to determining the other five $\mathcal{M}, \mathcal{N}, \mathcal{N}^{\prime}, \mathcal{P}$ and $\mathcal{Q}$. In Fig. 1, presented are the numerical results on the inertia parameters and the magnetic moments for various values of $m \mathcal{I}_{2}$ in the range $0 \leq m \mathcal{I}_{2} \leq 4$. The fitting process turns out to be remarkably independent of the parameter $m \mathcal{I}_{2}$; the inertia parameters $\mathcal{M}, \mathcal{P}$ and $\mathcal{N}+\frac{1}{2} \mathcal{N}^{\prime}$ are almost constants and the other parameters show a trivial linear dependence as

$$
\mathcal{N}+\frac{1}{2} \mathcal{N}^{\prime}=10.95, \quad m \mathcal{I}_{2} \mathcal{N}^{\prime}=5.06 m \mathcal{I}_{2}+2.87, \quad \text { and } \quad \mathcal{Q}=0.64 m \mathcal{I}_{2}-4.39
$$

Furthermore, for any value of $m \mathcal{I}_{2}$, the formula given in Table 1 can produce an excellent fit to the baryon magnetic moments with almost constant $\chi^{2}$ of order $10^{-3}$ and $F_{2, p}^{(s)}$ remains at a positive constant value about +0.4 . The numerical values are given in Table 2 as "Fit1".

Such an independence of the fitting to the parameter $m \mathcal{I}_{2}$ can be understood by using the V-spin symmetric relations (8). By subtracting a baryon magnetic moment by that

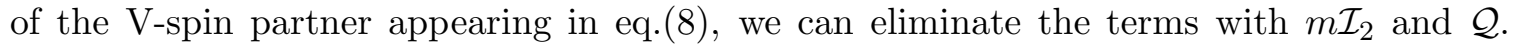
It leads us to three equations for $\mu_{p}-\mu_{\Xi^{-}}, \mu_{n}-\mu_{\Sigma^{-}}$and $\mu_{\Sigma^{+}}-\mu_{\Xi^{0}}$ for three unknowns $\mathcal{M}, \mathcal{N}+\frac{1}{2} \mathcal{N}^{\prime}$ and $\mathcal{P}$. With the experimental values for the baryon magnetic moments the equations can be easily solved as $\mathcal{M}=2.51, \mathcal{N}+\frac{1}{2} \mathcal{N}^{\prime}=10.76$ and $\mathcal{P}=-2.75$. These values are close to what we have obtained from the least-square fit including $\mu_{\Lambda}$. Once these three parameters are fixed, the remaining equation can be used to determine the rest three inertia parameters. However, we can get only two equations for three unknowns, $m \mathcal{I}_{2}, m \mathcal{I}_{2} \mathcal{N}^{\prime}$ and $\mathcal{Q}$, due to the last constraint in Eq.(\&), $\delta \mu_{2, \Sigma^{+}}=\frac{1}{2} \delta \mu_{2, p}$. By using $\mu_{p}$ and $\mu_{n}$ we obtain $m \mathcal{I}_{2} \mathcal{N}^{\prime}=4.96 m \mathcal{I}_{2}+3.34, \mathcal{Q}=0.64 m \mathcal{I}_{2}-4.91$, which are also comparable to Eq.(17). It explains the linear dependence of parameters $\mathcal{Q}$ and $m \mathcal{I}_{2} \mathcal{N}^{\prime}$ on $m \mathcal{I}_{2}$. The constraint leaves a relation for the magnetic moments as $\mu_{p}+\mu_{n}-\mu_{\Sigma^{-}}+\frac{1}{2} \mu_{\Xi^{-}}-\frac{3}{2}\left(\mu_{\Xi^{0}}+\mu_{\Sigma^{+}}\right)=0$. With the experimental magnetic moments the left hand side is evaluated as 0.08. It implies that the expressions given in Table 1 for the baryon magnetic moments are reasonable. The numerical results for this analysis are given as "Fit2" in Table 2, which yields again a positive strange form factor.

We present the numerical results of CBM 16] also in Table 2 as a reference. Here the inertia parameters are evaluated at the magic angle $\theta(R)=\frac{1}{2} \pi$ corresponding to the bag radius $R \sim 0.6 \mathrm{fm}$. The values are obtained by including the conventional Skyrme term to the meson part of the Lagrangian in order to stabilize the soliton solution with the Skyrme parameter $e=4.75$ and $f_{\pi}=93 \mathrm{MeV}$. The consequent baryon magnetic moments are presented in the next table, which show qualitative agreements with the experimental values within $30 \%$ errors. The worst case is the proton magnetic moment which comes out

\footnotetext{
${ }^{1}$ Ref. [15] has some erroneous numerical values in the predictions of the baryon magnetic moments and thus we use a more systematic approach.
} 
smaller than that of $\Sigma^{+}$. The same is true for the SM which corresponds to the CBM with zero bag radius.

Next we investigate the origin of the large positive values of the strange form factors. To do this we divide the nucleon strange form factors into three pieces; namely the contributions from the chiral symmetric limit $F_{2 N}^{(s), 0}$, the explicit FSB via the current operator $F_{2 N}^{(s), 1}$ and the implicit FSB through the representation mixing in the wave functions $F_{2 N}^{(s), 2}$ as shown in Table 3. Here one notes that the FSB effects are dominant in "Fit1", "Fit2" and the CBM through the explicit and implicit channels. This large contribution from the FSB originates from the terms which represent the differences in the kaon and the pion decay constants $f_{K} \neq f_{\pi}$ and in the masses $m_{K} \neq m_{\pi}$ and $m_{s} \neq m_{u, d}$. These terms implicitly describing the vacuum fluctuation in the strange direction have not been properly considered in most of models?. That is why our prediction in "Fit1", "Fit2" and the CBM are positively large differently from those of most of models.

In Table 3 one also notes that the explicit current FSB is dominant in "Fit1" and "Fit2" while the implicit representation mixing FSB is assertive in the CBM. Thus, one can hardly expect that those inertia parameters from the present state of chiral models could provide the ideal predictions of "Fit1" and "Fit2" on the magnetic moments and the strange form factors without an introduction of new idea

The up-, down- and strange-quark contributions to the magnetic moment of the proton are presented in Table 2. The results show that the up-quark contribution is dominant to those from down- and strange-quarks by a factor 10. It is fully consistent with the up-quark dominant picture of Ref. [8]. However, this qualitative behavior could not be used to predict positive strange form factor. Note that the I-spin symmetry leads us to $\mu_{p}^{(u, d, s)}$ as

$$
\mu_{p}^{(u)}=\frac{2}{3}\left[2 \mu_{p}+\mu_{n}+F_{2, p}^{(s)}(0)\right], \quad \mu_{p}^{(d)}=\frac{1}{3}\left[\mu_{p}+2 \mu_{n}+F_{2, p}^{(s)}(0)\right], \quad \text { and } \quad \mu_{p}^{(s)}=-\frac{1}{3} F_{2, p}^{(s)} .
$$

which would yield $\mu_{p}^{(u)}=2.45$ n.m. and $\mu_{p}^{(d)}=-0.34$ n.m. in case of vanishing strange form factor. Our theoretical prediction for the strange form factor of the other baryons is also given in Table 2 .

In summary, we have investigated the magnetic moments and the strange form factors of the baryon octet based on the group structure of the chiral models. Various symmetry breaking terms are included into the model and treated as perturbations in the $\mathrm{SU}(3)$ collective coordinate quantization scheme. Six inertia parameters appearing in the formulas for the magnetic moments are adjusted to fit the experimental values of the baryon octet. Our formulas are general to all the soliton models for the baryons with the SU(3) symmetry breaking terms treated as a perturbation. We expect that in a more sophisticated version of CBM the situation is improved. The formulas turn out to fit the baryon magnetic moment remarkably well and predict the positive strange form factor of the proton $0.37 \mathrm{n} . \mathrm{m}$. to

\footnotetext{
${ }^{2}$ Here one notes that one reference $[10]$ treats the FSB effects to yield $F_{2, p}^{(s)}=-0.13$ n.m.. However this value has the same sign but is much larger than our SM prediction due to the fact that they used the different Skyrme parameter $e=4.0$ and missed in the inertia parameter $m$ the contribution from the term proportional to $f_{K}^{2}-f_{\pi}^{2}$.

${ }^{3}$ Here one cannot exclude the possibility of introducing new additional inertia parameters in the model independent relations which could yield a different prediction of the strange form factors, together with the gluon and Casimir effects.
} 
be compared with the recent experimental result $0.23 \pm 0.37 \pm 0.15 \pm 0.19$ n.m. [2]. We emphasize the role of the vacuum fluctuation in the strange direction to give the positive result, which is ignored in most of models.

We would like to thank Mannque Rho and G.E. Brown for helpful discussions and constant concerns. This work is supported in part by the Korea Science and Engineering Foundation through the CTP and by the Korea Ministry of Education under Grant No. BSRI-97-2418.

\section{References}

[1] J. Ashman et al., Phys. Lett. B206 (1988) 364.

[2] B. Mueller et al., Phys. Rev. Lett. 78 (1997) 3824.

[3] R. D. McKeown, Phys. Lett. B219 (1989) 140; E.J. Beise and R.D. McKeown, Comm. Nucl. Part. Phys. 20 (1991) 105.

[4] D.B. Kaplan and A.E. Nelson, Phys. Lett. B175 (1986) 273.

[5] C.H. Lee, G.E. Brown, D.P. Min and M. Rho, Nucl. Phys. A585 (1995) 401.

[6] A.E. Nelson and D.B. Kaplan, Phys. Lett. B192 (1987) 273.

[7] G.E. Brown, K. Kubodera, D. Page and P. Pizzochero, Phys. Rev. D37 (1988) 2042.

[8] R.D. McKeown, Los Alamos Preprint hep-ph/9607340.

[9] R.L. Jaffe, Phys. Lett. B229 (1989) 275.

[10] N.W. Park, J. Schechter and H. Weigel, Phys. Rev. D43 (1991) 869.

[11] W. Koepf, E. M. Henley and S.J. Pollock, Phys. Lett. B288 (1992) 11.

[12] M.J. Musolf and M. Burkardt, Z. Phys. C61 (1994) 433.

[13] S.C. Phatak and S. Sabu, Phys. Lett. B321 (1994) 11.

[14] C.V. Christov et al., Prog. Part. Nucl. Phys. 37 (1996) 1.

[15] S.T. Hong and B.Y. Park, Nucl. Phys. A561 (1993) 525.

[16] B.Y. Park and M. Rho, Z. Phys. A331 (1988) 151.

[17] Ulf-G. Meissner et al., Los Alamos Preprint hep-ph/9701296.

[18] G.S. Adkins, Solitons in Nuclear and Elementary Particle Physics, p47, World Scientific (1984), Editors: A. Chodos, E. Hadjimichael and C. Tze.

[19] B.Y. Park, D.P. Min and M. Rho, Nucl. Phys. A517 (1990) 561. 
Table 1: Electromagnetic moments of baryon octet : expressions.

\begin{tabular}{lccccc}
\hline$\mu_{B}=$ & $\mu_{0, B}$ & $\oplus$ & $\delta \mu_{1, B}$ & $\oplus$ & $\delta \mu_{2, B}$ \\
\hline$\mu_{p}=$ & $+\frac{4}{40} \mathcal{M}+\frac{8}{30}\left(\mathcal{N}+\frac{1}{2} \mathcal{N}^{\prime}\right) \oplus+\frac{16}{90} \mathcal{P}-\frac{8}{180} \mathcal{Q} \oplus$ & $m \mathcal{I}_{2}\left[\frac{24}{1500} \mathcal{M}+\frac{16}{2250}\left(\mathcal{N}-2 \mathcal{N}^{\prime}\right)\right]$ \\
$\mu_{n}=$ & $+\frac{2}{40} \mathcal{M}-\frac{6}{30}\left(\mathcal{N}+\frac{1}{2} \mathcal{N}^{\prime}\right) \oplus-\frac{10}{90} \mathcal{P}+\frac{14}{180} \mathcal{Q} \oplus m \mathcal{I}_{2}\left[\frac{62}{1500} \mathcal{M}-\frac{92}{2250}\left(\mathcal{N}-\frac{21}{23} \mathcal{N}^{\prime}\right)\right]$ \\
$\mu_{\Lambda}=$ & $+\frac{1}{40} \mathcal{M}-\frac{3}{30}\left(\mathcal{N}+\frac{1}{2} \mathcal{N}^{\prime}\right) \oplus-\frac{9}{90} \mathcal{P}-\frac{9}{180} \mathcal{Q} \oplus$ & $m \mathcal{I}_{2}\left[\frac{27}{1500} \mathcal{M}+\frac{18}{2250}\left(\mathcal{N}-2 \mathcal{N}^{\prime}\right)\right]$ \\
$\mu_{\Xi^{0}}=$ & $+\frac{2}{40} \mathcal{M}-\frac{6}{30}\left(\mathcal{N}+\frac{1}{2} \mathcal{N}^{\prime}\right) \oplus-\frac{22}{90} \mathcal{P}-\frac{4}{180} \mathcal{Q} \oplus$ & $m \mathcal{I}_{2}\left[\frac{12}{1500} \mathcal{M}+\frac{8}{2250}\left(\mathcal{N}-2 \mathcal{N}^{\prime}\right)\right]$ \\
$\mu_{\Xi^{-}}=$ & $-\frac{6}{40} \mathcal{M}-\frac{2}{30}\left(\mathcal{N}+\frac{1}{2} \mathcal{N}^{\prime}\right) \oplus-\frac{8}{90} \mathcal{P}-\frac{8}{180} \mathcal{Q} \oplus m \mathcal{I}_{2}\left[\frac{24}{1500} \mathcal{M}+\frac{16}{2250}\left(\mathcal{N}-2 \mathcal{N}^{\prime}\right)\right]$ \\
$\mu_{\Sigma^{+}}=$ & $+\frac{4}{40} \mathcal{M}+\frac{8}{30}\left(\mathcal{N}+\frac{1}{2} \mathcal{N}^{\prime}\right) \oplus+\frac{26}{90} \mathcal{P}-\frac{4}{180} \mathcal{Q} \oplus m \mathcal{I}_{2}\left(\frac{12}{1500} \mathcal{M}+\frac{8}{2250}\left(\mathcal{N}-2 \mathcal{N}^{\prime}\right)\right]$ \\
$\mu_{\Sigma^{-}}=$ & $-\frac{6}{40} \mathcal{M}-\frac{2}{30}\left(\mathcal{N}+\frac{1}{2} \mathcal{N}^{\prime}\right) \oplus-\frac{4}{90} \mathcal{P}+\frac{14}{180} \mathcal{Q} \oplus m \mathcal{I}_{2}\left[\frac{62}{1500} \mathcal{M}-\frac{92}{2250}\left(\mathcal{N}-\frac{21}{23} \mathcal{N}^{\prime}\right)\right]$ \\
$\mu_{\Sigma^{0}}=$ & $-\frac{1}{40} \mathcal{M}+\frac{3}{30}\left(\mathcal{N}+\frac{1}{2} \mathcal{N}^{\prime}\right) \oplus+\frac{11}{90} \mathcal{P}+\frac{5}{180} \mathcal{Q} \oplus m \mathcal{I}_{2}\left[\frac{37}{1500} \mathcal{M}-\frac{42}{2250}\left(\mathcal{N}-\frac{17}{21} \mathcal{N}^{\prime}\right)\right]$ \\
\hline$\mu_{N}^{(s)}=$ & $-\frac{7}{60} \mathcal{M}+\frac{1}{45}\left(\mathcal{N}+\frac{1}{2} \mathcal{N}^{\prime}\right) \oplus$ & $+\frac{1}{45} \mathcal{P}+\frac{1}{90} \mathcal{Q} \oplus m \mathcal{I}_{2}\left[\frac{43}{2250} \mathcal{M}-\frac{38}{3375} \mathcal{N}+\frac{26}{3375} \mathcal{N}^{\prime}\right]$ \\
$\mu_{\Lambda}^{(s)}=$ & $-\frac{3}{20} \mathcal{M}-\frac{1}{15}\left(\mathcal{N}+\frac{1}{2} \mathcal{N}^{\prime}\right) \oplus-\frac{1}{15} \mathcal{P}-\frac{1}{30} \mathcal{Q} \oplus$ & $m \mathcal{I}_{2}\left[\frac{3}{250} \mathcal{M}+\frac{2}{1125} \mathcal{N}-\frac{4}{375} \mathcal{N}^{\prime}\right]$ \\
$\mu_{\Xi}^{(s)}=$ & $-\frac{1}{5} \mathcal{M}-\frac{4}{45}\left(\mathcal{N}+\frac{1}{2} \mathcal{N}^{\prime}\right) \oplus$ & $-\frac{1}{9} \mathcal{P}-\frac{1}{45} \mathcal{Q} \oplus$ & $m \mathcal{I}_{2}\left[\frac{1}{125} \mathcal{M}+\frac{4}{1125} \mathcal{N}-\frac{8}{1125} \mathcal{N}^{\prime}\right]$ \\
$\mu_{\Sigma}^{(s)}=$ & $-\frac{11}{60} \mathcal{M}+\frac{1}{15}\left(\mathcal{N}+\frac{1}{2} \mathcal{N}^{\prime}\right) \oplus+\frac{11}{135} \mathcal{P}+\frac{1}{54} \mathcal{Q} \oplus m \mathcal{I}_{2}\left[\frac{37}{2250} \mathcal{M}-\frac{14}{1125} \mathcal{N}+\frac{34}{3375} \mathcal{N}^{\prime}\right]$ \\
\hline
\end{tabular}


Table 2: The inertia parameters, magnetic moments of baryon octet and their form factors. The magnetic moments included in the fitting process are indicated by $*$. For the inertia parameters for "Fit1", see the discussions in the text and Eq. (17).

\begin{tabular}{ccccccc}
\hline & $\mathcal{M}$ & $\mathcal{N}$ & $\mathcal{N}^{\prime}$ & $\mathcal{P}$ & $\mathcal{Q}$ & $m \mathcal{I}_{2}$ \\
\hline Fit1 & 2.55 & text & text & -2.91 & text & free \\
Fit2 & 2.51 & 7.73 & +6.07 & -2.75 & -2.99 & 3.00 \\
CBM & 0.66 & 6.00 & +0.52 & +1.11 & +1.27 & 3.96 \\
SM & 0.67 & 5.03 & +0.91 & +0.76 & +0.99 & 1.79 \\
\hline
\end{tabular}

\begin{tabular}{ccccccccc}
\hline & $\mu_{p}$ & $\mu_{n}$ & $\mu_{\Lambda}$ & $\mu_{\Xi^{0}}$ & $\mu_{\Xi^{-}}$ & $\mu_{\Sigma^{+}}$ & $\mu_{\Sigma^{0}}$ & $\mu_{\Sigma^{-}}$ \\
\hline Fit1 & $2.80^{*}$ & $-1.91^{*}$ & $-0.58^{*}$ & $-1.28^{*}$ & $-0.71^{*}$ & $2.40^{*}$ & 0.62 & $-1.16^{*}$ \\
Fit2 & $2.79^{*}$ & $-1.91^{*}$ & $-0.56^{*}$ & $-1.27^{*}$ & $-0.69^{*}$ & $2.41^{*}$ & 0.62 & $-1.16^{*}$ \\
CBM & 2.06 & -2.03 & -0.58 & -1.43 & -0.49 & 2.12 & 0.43 & -1.25 \\
SM & 1.68 & -1.33 & -0.59 & -1.24 & -0.52 & 1.76 & 0.54 & -0.68 \\
\hline Exp. & 2.79 & -1.91 & -0.61 & -1.25 & -0.69 & 2.43 & - & -1.16 \\
\hline
\end{tabular}

\begin{tabular}{ccccccccc}
\hline & $\mu_{p}^{(u)}$ & $\mu_{p}^{(d)}$ & $\mu_{p}^{(s)}$ & \multicolumn{1}{c}{$F_{2 N}^{(s)}$} & $F_{2 \Lambda}^{(s)}$ & $F_{2 \Xi}^{(s)}$ & $F_{2 \Sigma}^{(s)}$ & \multicolumn{1}{c}{$F_{2}^{0}$} \\
\hline Fit1 & 2.72 & 0.21 & -0.13 & 0.39 & 1.43 & 1.26 & -0.97 & 0.28 \\
Fit2 & 2.70 & 0.22 & -0.12 & 0.37 & 1.37 & 1.22 & -0.99 & 0.26 \\
CBM & 1.59 & 0.57 & -0.10 & 0.30 & 0.49 & 0.25 & -1.54 & -0.67 \\
SM & 1.34 & 0.33 & +0.01 & -0.02 & 0.51 & 0.09 & -1.74 & -0.67 \\
\hline
\end{tabular}

Table 3: The strange form factors of nucleon.

\begin{tabular}{crrrr}
\hline & $F_{2 N}^{(s), 0}$ & $\delta F_{2 N}^{(s), 1}$ & \multicolumn{1}{c}{$\delta F_{2 N}^{(s), 2}$} & $F_{2 N}^{(s)}$ \\
\hline Fit1 & 0.16 & 0.28 & -0.05 & 0.39 \\
Fit2 & 0.16 & 0.28 & -0.07 & 0.37 \\
CBM & -0.19 & -0.12 & 0.61 & 0.30 \\
SM & -0.13 & -0.09 & 0.20 & -0.02 \\
\hline
\end{tabular}


Figure 1: Numerical results of the fitting : (a) inertia parameters, (b) $\chi^{2}$, (c) baryon magnetic moments and (d) proton strange form factor as a function of $m \mathcal{I}_{2}$. The solid lines in (c) are the experimental values of the baryon magnetic moments.

(a)

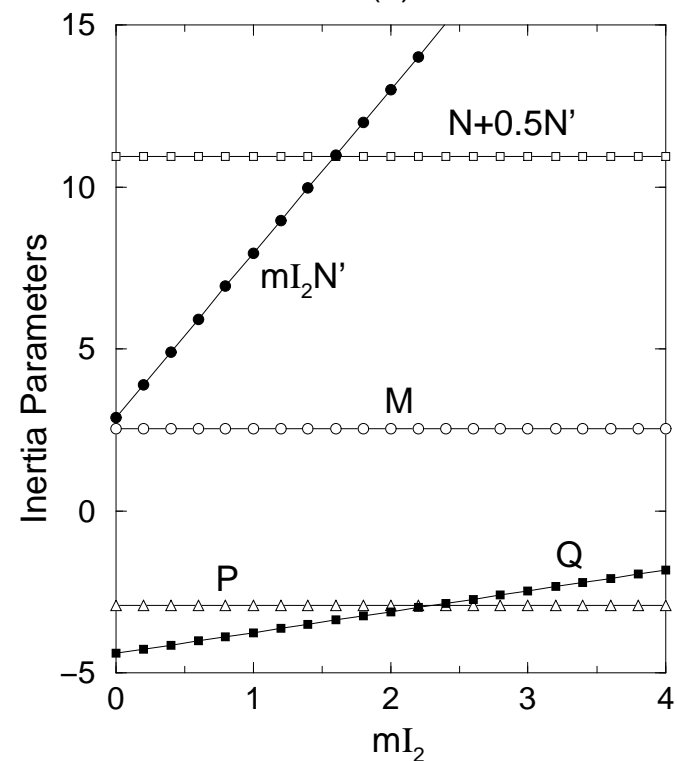

(b)

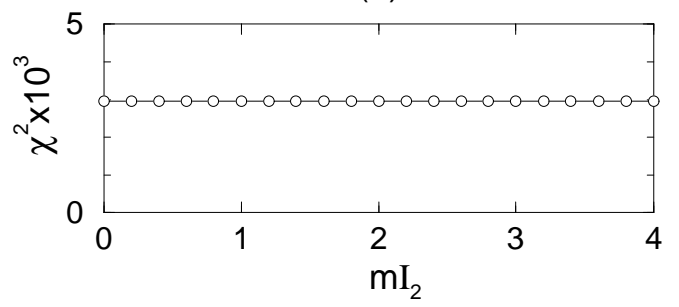

(c)

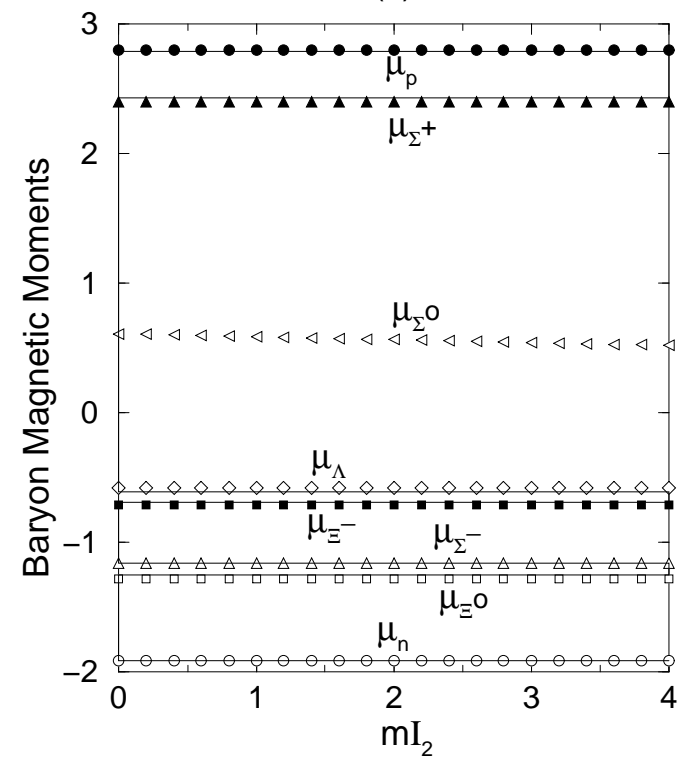

(d)

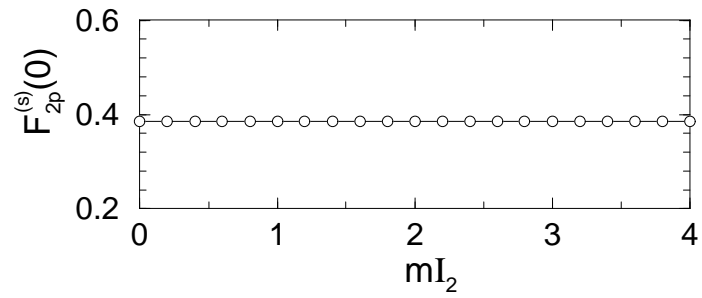

